\title{
COMPARISON OF GONIOMETRIC MEASUREMENT RELIABILITY OF HIP JOINT FLEXION AND PROXIMAL INTERPHALANGEAL JOINT FLEXION IN HEALTHY AdULTS
}

\begin{abstract}
SUMMARY: Background For many reasons physiotherapists measure joint range of motion of their patients. There are usually variations in measurement values of each motion when measured either by the same person or by different observers. Also reliability studies have resulted in varying levels of reliability

\section{OKEKE H, MA, MCSP, PT}

Physical Therapy Department, Kuwait University coefficients. The purpose of this study was to compare the goniometric measurement reliability of a complex joint represented by the hip joint and a simple joint represented by proximal interphalangeal (PIP) flexion motions and to assess whether the two joint motions could be equally reliably measured by the same person under the same test and retest conditions. Method Thirty-three healthy subjects between the ages of 23 and 34 years participated. In order to assess the repeatability of measurements in the two selected joint motions, the r. level of each joint measurement was determined and their goniometric measurement reliability compared. Result Results were based on data collected by measuring the flexion range of motion of right hips and right middle finger PIP joints in normal adults. Statistical analyses indicated that there were significant differences between goniometric measurement reliability in hip and PIP flexion motions. Conclusion Two joints were goniometrically measured by the same tester under the same test condition. Reliability measurements results were compared in thirty-three adults. Based on the study, the researcher concluded that the goniometric measurement of hip flexion motion is more variable than that of PIP flexion motion.
\end{abstract}

\section{KEYWORDS: JOINT-MEASUREMENT-RELIABILITY, HIP-JOINT, PIP-JOINT, GONIOMETRY.}

\section{INTRODUCTION}

Joint motion measurement is used as part of techniques for arriving at diagnosis, assessing progress of treatment, clinical estimation of permanent disabilities as well as providing records in future comparison (Cole \& Tobis, 1982). The importance of joint measurement in legal determination of disability and provision of research data cannot be underestimated (Gajdosik \& Bohannor, 1987). Clinicians measure range of motions of their patients not only to determine baseline limitations before actual treatment commences, but also to assess

\section{CORRESPONDENCE:}

\section{HA Okeke}

Faculty of Allied Health Sciences Physical Therapy Department School of Allied Health Professions Kuwait University

P O Box 31470

90805 - Sulaibikhat KUWAIT

Tel: (965) 482-4325 (H)

(965) 483-3706 (W)

Fax:(065) 483-0937

E-mail: Okeke@hsc.kuniv.edu.kw the efficacy of intervention employed. Many different devices have been used generally for range of motion measurements such as estimation (Somers et al, 1997), photography (Miller, 1985), xerography (Regenos \& Chyatle, 1970). Also specific joint measurement devices and methods have been used to assess range of motion of specific joints (Nwaobi, 1987).

Hamilton and Lachenbruch, (1969) compared different types of goniometers - specific dorsal, pendulum and universal for assessment of finger joint angles and found that the three goniometric types were equally reliable. Rothstein et al, (1983) found no difference in reliability of large metal, large plastic and small universal goniometers. Goniometer is by for the most popular device for measuring joint range and commonly used goniometer by many clinicians is the Universal Goniometer (UG) whose validity and reliability have been accepted. For this study, a small metal universal goniometer calibrated in degrees was used.

Miller (1985) reported normal values of range of motion of major joints of the body from ten referenced-sources. The values for the elbow flexion ranged from $135^{\circ}-160^{\circ}$ (mean $146.8^{\circ}$ ), hip flexion from $110^{\circ}-130^{\circ}$ (mean $119.7^{\circ}$ ) and hip abduction from $45^{\circ}-55^{\circ}$ (mean $46.1^{\circ}$ ) The standard deviations of measurement errors for the three motions were elbow $8.7^{\circ}$, hip flexion $7.6^{\circ}$ and hip abduction $3.9^{\circ}$. Thus, there was evidence of levels of variations in measurement errors in each of the three joint motions. Those variations existed not only in the normal values of each motion in the ten different studies but also in the level of dif ferences between one joint motion and the other.

Although most joints of the body have been shown both in patients and healthy subjects as being capable of being reliably measured, reliability studies have resulted in varying levels of reliability coefficient (Babyar, 1996; Youdas et al, 1994). The purpose of this study, therefore, was (1) to determine whether some joint motions could be more reliably measured than others, in particular (1.1) to assess the goniometric measurement reliability of the hip joint and proximal interphalangeal (PIP) joint flexion 
motions in normal subjects and (1.2) to determine whether the two joint motions could be measured with high levels of reliability by the same tester under the same test conditions using the UG.

The study was considered important because as much as goniometric reliability of joint measurements enable clinicians to detect variations in joint range, variations may be consistently more in some joints than in others. Prior knowledge of range of motion characteristics of joint types would enable judicious decisions to be made regarding the nature of interventions. The choice of hip and PIP joints was based on the understanding that the hip was more complex a joint than PIP joint. Flexion motions in the two joints run through identical excursion of $180^{\circ}-0^{\circ}$ using that system of notation so that any difference in reliability measurements under the same type of control in healthy subjects must not be due to measurement errors. The researcher hypothesized that there was no difference between goniometric range of motion measurement reliability in the hip joint flexion and goniometric range of motion measurement reliability in the PIP joint flexion motion.

Earlier studies based on goniometric joint measurements repeated by the same person or by different observers found high levels of goniometric reliability in measurements. However, Hellebrandt et al, (1948) did not find wrist flexion or shoulder abduction measurements reliable when repeatedly measured. Determination of goniometric reliability of hip measurements (Gajdosik et al, 1985) and that of finger joints (Serup, 1983) have also been conducted. However, the studies encompassed only tester, instrument and condition variables. Gajdosik \& Bohannor, (1987) reviewed literature on reliability and validity of goniometric measurements of extremities. Based on the conclusions of earlier studies (Gajdosik et al, 1985) they concluded that hip motions could be reliably measured if measurement procedures were properly controlled. The implication is that variability in range of motion measurement reliability could be eliminated in any joint provided the same procedural control was observed no matter the type of joint.

\section{METHOD}

Subjects:

Thirty-three healthy volunteer physical therapists, physical therapy interns and final year physical therapy students were selected from the Physical Therapy Department of University of Nigeria Teaching Hospital, Enugu, Nigeria to participate in the study. The participants, eighteen women and fifteen men were screened for hand preference and history of injury or illness that might affect range of motions of the joints of interest. They were also told to report any pathology which might affect the range of motion between the initial measurement and subsequent measurements. Written informed consent was obtained from all the participants before participation in the study. Demographic information of the subjects are summarised in Table 1.

\section{TABLE 1: CHARACTERISTICS OF SUBJECTS}

\begin{tabular}{|l|l|}
\hline Number of subjects & 33 \\
\hline Female & 18 \\
\hline Male & 15 \\
\hline Age(years) & \\
\hline$x$ & 28.1 \\
\hline SD & 3.4 \\
\hline Range & $23-34$ \\
\hline
\end{tabular}

\section{Instrument}

A small metal half-cycle protractor scalestype UG was used to measure the range of motions of the joints of interest in degrees. The stationary and movable arms were each $14.5 \mathrm{~cm}$ in length from the axis (Fig. 1). The choice of UG was determined by its obvious advantages

\section{FIGURE 1:COMPARISON OF HIP AND PIP FLEXION MEASUREMENT RELIABILITY}

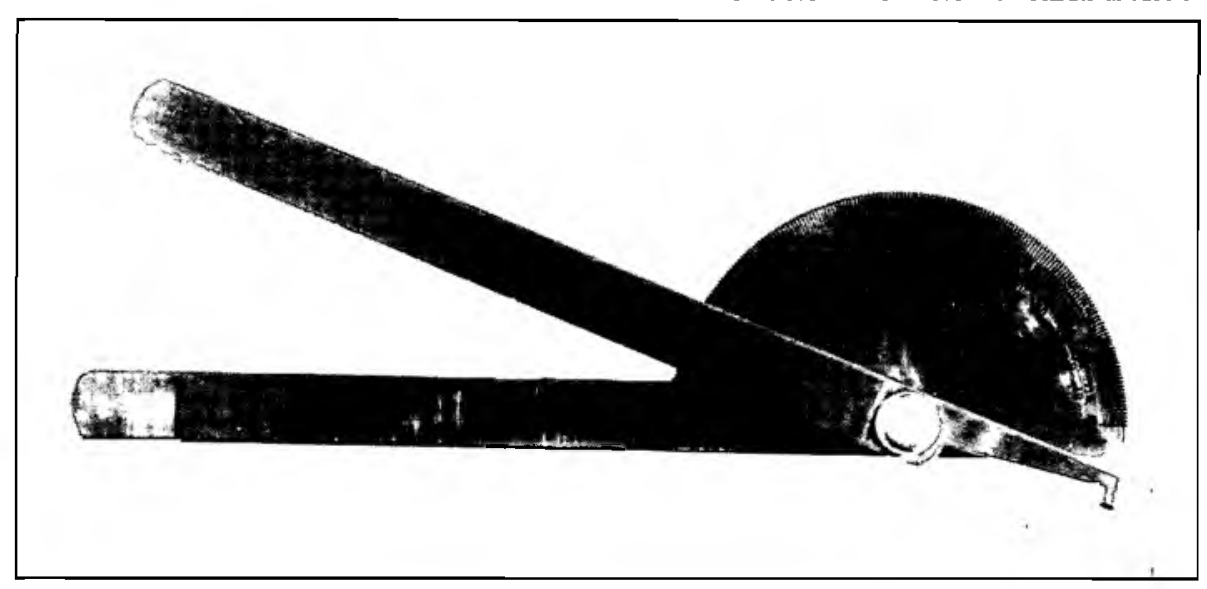

including that it could be used to measure many types and sizes of joints. It is considered to be commonly used clinically being the simplest, most portable and easily available. Moreover, UG having been established as an instrument for measurement of range of motion in criterion measure for determining the validity of new instruments designed for measurement of joint range.

\section{Procedure}

The procedure for measurement of hip flexion motion was a modification on procedures employed by earlier workers. The subject was supine with hip and knee of the side to be measured in extension on a flat surface. To determine the axis of the right hip, a line joining the right anterior superior iliac spine and symphysis pubis was bisected by a line running laterally to the right greater trochanter. The axis of the goniometer was then placed on the external point corresponding to the axis of the hip joint. The stationary arm was aligned with the lateral side of the pelvis while the movable arm was placed lateral to the long axis of the femur. The subject was then asked to bend the left hip and knee as far as they could go. The movable arm of the goniometer was held against the thigh with which it moved as the right leg was being raised. At the limit of flexion of the hip, the reading on the goniometer was recorded.

To measure the PIP joint whose flexion motion is functionally dependent on the influence of adjacent proximal and distal joints to it, a special stabilizing device was used to immobilize other degrees, has frequently been used as 
FIGURE 2:COMPARISON OF HIP AND PIP FLEXION MEASUREMENT RELIABILITY

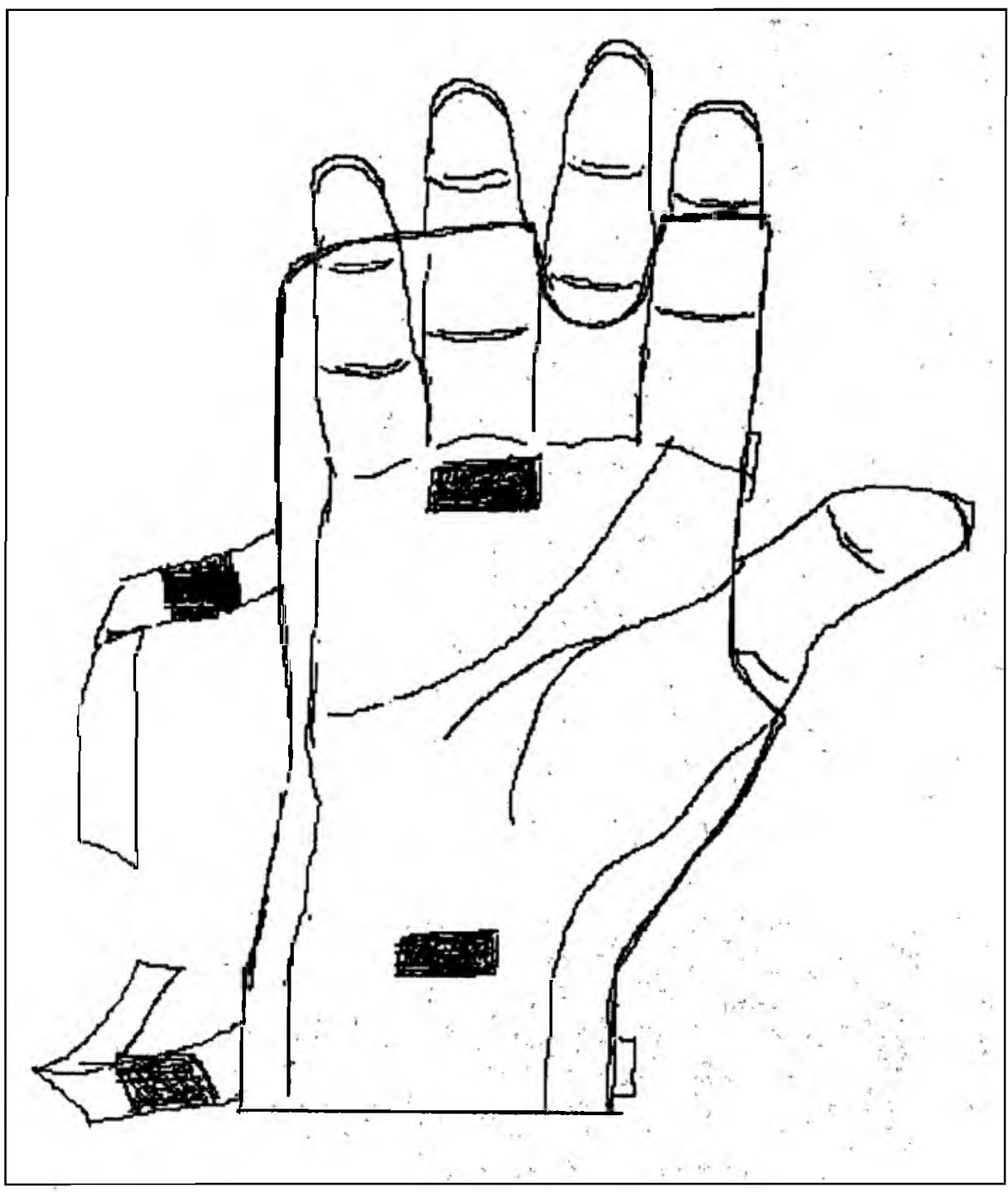

nearby joint movements from the wrist, hand and finger other than the desired flexion motion of PIP joint of the right middle finger (Fig. 2). With the subject sitting, the right forearm was supinated and with the stabilizing device secured, the axis of the goniometer was placed at the ulnar side of the axis of rotation of the joint. The stationary arm was placed to run along the proximal bone and movable arm along the digital bone. The subject was asked to bend the finger and at its limit of flexion, the goniometer reading was taken. Common to procedures in the two joints were:

a) The range of motions was active

b) The two motions were carried out against gravity.

c) Each measurement was repeated three times and the average found and recorded as the score for that measurement session. d) Positioning was rigidly standardized during measurement.

e) The $0^{\circ}-180^{\circ}$ system of notation was used.

All measurement data was collected between the second week of July and second week of August, 1997. Measurement sequence was alternated between the two joints.

\section{Data Analysis}

Obtained data was analyzed by descriptive statistical procedures relating to mean, standard deviation and coefficient of variation. Pearson product-moment correlation coefficient was employed to analyse the reliability of measurements of the two joints flexion motions. Finally, analysis of variance (ANOVA) was used to test the hypothesis that there was no difference between the goniometric range of motion measure- ment reliability in the hip joint flexion and goniometric range of motion measurement reliability in the PIP joint flexion motion.

\section{RESULTS}

The average of three measurements was computed to represent the score for each session. Table 2 summarizes the highlights of descriptive statistical data obtained from the test and the retest sessions for each joint.

To compare the test and retest measurements of each of the two joints, Pearson Product-moment correlation coefficient for intratester reliability yielded $r=0.93$ for hip flexion and $r=0.99$ for PIP joint flexion. Coefficient of variation $(\mathrm{CV})$ for hip was $11.8 \%$ and for PIP $8.8 \%$. Finally ANOVA indicated that there was a difference between goniometric measurement reliability in hip and PIP flexion motions.

The results suggested that PIP joint flexion motion was more reliably measured than the hip joint flexion motion in normal adults.

\section{DISCUSSION}

The study was designed to determine the comparative reliability of goniometric measurements of a complex joint - the hip, and a simple hinge joint - PIP joint flexion motions. The results obtained from statistical analysis allow for rejection of null hypothesis.

The researcher obtained high correlation coefficients for both hip and PIP joint flexion motions viz $\mathrm{r}=0.93$ and $r=0.99(p<0.001)$ respectively. Although the design of the study was not intended primarily to test measurement precision, the high correlation values in each joint had two implications. Firstly, the coefficient values form part of the data on which comparisons of the two joint motions could be based. Secondly, high correlation coefficient values gave further assurance that measurement error was minimized indicating that the components of the test environment and methods were properly controlled.

The Coefficient of Variation of hip flexion motion was higher than that of PIP flexion motion. The differences in the coefficient of variation indicated the relative variation of hip flexion motion 
TABLE 2: GONIOMETRIC MEASUREMENT IN THE HIP AND PIP ACTIVE FLEXION MOTION

\begin{tabular}{|l|l|l|}
\hline \multicolumn{1}{|c|}{ HIP } & \multicolumn{1}{c|}{ MEAN } & \multicolumn{1}{c|}{ SD } \\
\hline Measurement 1 & 105.76 & 12.76 \\
\hline Measurement 2 & 104.38 & 12.02 \\
\hline PIP & & \\
\hline Measurement 1 & 117.76 & 10.39 \\
\hline Measurement 2 & 118.48 & 10.49 \\
\hline
\end{tabular}

and PIP flexion motion measurements. These differences helped to remove any doubt that might have existed in interpretation of the differences in their means and standard deviations. Finally, based on ANOVA conducted on the two joint measurement data, the hip joint flexion motion was significantly less reliably measured than that of PIP.

This study contrasted with other reported studies relating to goniometric reliability measurements done in the past. The use of present methodology to compare the reliability of goniometric measurements of two anatomically dissimilar joints has not previously appeared in the literature. The shoulder and hip as complex joints have three degrees freedom of movement. In contrast, the elbow, knee and PIP joints are classified as hinge joints with one degree freedom of movement. Difficulties in determining measurement reliability of complex joints have been recognized (Gajdosik \& Bohannor, 1987). Many reasons have been suggested for difficulty in measurement of complex joints. These include presence of two joint muscles whose actions varied according to situations (Rothstein et al, 1983), bulk of muscles around them which often limited full movement excursion and concurrent movement of adjacent joints (Gajdosik et al, 1985).

However, Boone et al, (1978) compared goniometric reliability of three joints of the upper extremity with three joints of lower extremity. Their findings implied, amongst others, that two complex joints - shoulder and hip motions were more reliably measured $(r=0.96$ and 0.76 respectively) than those of two simple hinge joints - elbow and knee ( $r=0.94$ and 0.74 respectively). The results may have been influenced by the fact that the researchers focused attention on comparison between the upper and lower extremity joints rather than with the types and behaviors of individual joints making up the groups.

Ekstrand et al, (1982) in a study to determine the reliability of goniometric measurements of the joints of the lower extremity found measurement of hip joint flexion more variable than that of knee joint flexion. Rothstein et al, (1983) assessed intratester reliability of goniometric measurements of passive elbow and knee joint flexions using three types of goniometers. Pearson Product moment correlation coefficient values in the two joints were high and showed $r$ of 0.96 and $\mathrm{r}=0.98$ for elbow and knee respectively. The findings of the above two studies were in line with those of the present study.

Also, Miller, (1985) reported the raw data of hip flexion measurements from ten referenced-sources and based on the data, the present researcher calculated the $\mathrm{CV}$ of flexion motions for the shoulder and hip as complex joints as well as for the elbow and knee as simple hinge joints. The results showed more variability in the shoulder and hip (CV 18.1\% and $7.3 \%$ respectively) compared with variability in the elbow and knee (CV $5.2 \%$ and $5.3 \%$ respectively). These findings derived from Miller's report were also in agreement with the outcome of the present study.

The choice of hip flexion motion has been considered apt by the researcher because of common occurrences of hip flexion contractures in neurologic, orthopaedic and other situations of inactivity as often seen in the elderly population. The need for clinicians to appreciate the limits of variability in hip flexion motion cannot, therefore, be overstressed.

Little has been reported on PIP joint of the hand. Neverthless, it is an important functional joint of the hand whose predictive range clinicians ought to be conversant with. Oedema and pain tend to place the PIP in flexion which often makes flexion deformity of the joint a very common feature. Its intermediate position in a segmented lever (finger) makes it possible for objects to be firmly gripped. When the range of motion of PIP joint is limited such as in swan neck deformity, boutonniere deformity or as a result of adhesion of soft tissues around the joint following trauma, smooth and relay buckling of the finger into the palm becomes compromised and crippling.

Active range of motion of joints is primarily related to the degree to which ligaments and muscles function. In case of hip and PIP joints, superimposition of pathologies make their functions difficult to assess. Understanding the peculiarities of measurement behaviours of each joint of the body, is important, because the clinician is in a position to discern between restrictions imposed by pathology and limitations due to inherent idiosyncracy of the joint. However, this study should serve as a spring-board for further studies to determine whether such differences in reliability measurements of many other anatomically dissimilar joints exist. Also many more subjects both patients and healthy subjects under varying environmental conditions should be involved.

\section{CONCLUSION}

Two joints were goniometrically measured by the same tester under the same test condition. Reliability measurements results were compared in 33 healthy adults. Based on the study, the researcher concluded that the goniometric measurement of hip flexion motion is more variable than that of the PIP flexion motion. In research and clinical settings where reliable measurements are essential, this study provides further understanding of goniometric measurements applied to two particular joints by a single therapist. The results can only be generalized to healthy and relatively young subjects. 
1. Babyar SR 1996 Reliability of student physical therapists in performing two techniques for goniometric measurement of passive shoulder position in the horizontal plane. Journal of Physical Therapy Education 10:85-89

2. Boone DC, Azen SP 1979 Normal range of motion of joint in male subjects. Joumal of Bone and Joint Surgery 61:156-159

3. Cole TM, Tobis JS 1982 Measurement of musculoskeletal function. In Kottke FJ, Stillwell GK, Lehann JF (eds). Krusen's Handbook of Physical Medicine and Rehabilitation. Philadelphia. W.B. Saunders Co., Philadelphia

4. Ekstrand J, Wiktorsson M, Oberg B 1892 Lower extremity goniometric measurements: A study to determine their reliability. Archives of Physical Medicine and Rehabilitation 63:171-175

5. Gajdosik RL, Bohannon RW 1987 Clinical measurement of range of motion: Review of goniometry emphasizing reliability and validity. Physical Therapy 67:1867-1872
6. Gajdosik RL, Simpson R, Smith R, Dantiony R 1985 Pelvic tilt: Intratester reliability of measuring the standing position and range of motion. Physical Therapy 65:169174

7. Hamilton GF, Lachenbruch PA 1969 Reliability of goniometers in assessing finger joint angle. Physical Therapy 49:465-469

8. Hellebrandt FA, Duvall EN, Moore ML 1948 The measurement of joint motion part III: Reliability of goniometry. Physical Therapy Review 29:302-307

9. Miller PJ 1985 Assessment of joint motion In Rothstein (ed) Measurement in Physical Therapy. Churchill Livingstone, New York

10. Nwaobi OM 1987 Goniometer system for measuring bilateral hip abduction in cerebral palsy: Suggestion from the field. Physical Therapy 67:953-954

11. Regenos EM, Chyatle SB 1970 Joint range and deformity recorded by xerography. Physical Therapy 50:190
12. Rothstein JM, Miller PJ, Roettger RF 1983 Elbow and knee measurements. Physical Therapy 63:1611-1615

13. Serup J 1983 Measurement of contractures of digits in systemic sclerosis. Dermatologia 167:250-255

14. Somers DL, Hanson JA, Kedzierski CM, Nestor KL, Quinlivan KY 1997 The influence of experience on the reliability of goniometric and visual measurement of forefool position. Journal of Orthopaedic Sports Physical Therapy 25: 192-302

15. Youdas JW, Carey JR, Garrett TR, Suman VJ 1994 Reliability of goniometric measurements of active arm elevation in the scapular plane obtained in a clinical setting. Archives of Physical Medicine and Rehabilitation 75: 1137-1144

\section{THE SOUTH AFRICAN SOCIETY OF PHYSIOTHERAPY} would like to congratulate Prof. Sielie Eales on her appointment as Editor of the S.A. Journal of Physiotherapy. We know she will bring a wealth of experience in various sectors, to the position.

We would also like to thank Prof. Muriel Goodman, outgoing Editor, for her achievements since being at the helm and wish her much peace and happiness in her retirement. 